\title{
Statistical Ranging of Orbits for Trans-Neptunian Objects
}

\author{
J. Virtanen, K. Muinonen \\ Observatory, Univ. of Helsinki, P.O.Box 14, FIN-00014 Univ. of \\ Helsinki, Finland \\ E. Bowell \\ Lowell Observatory, 1400 W. Mars Hill Road, Flagstaff, Arizona 86001
}

\begin{abstract}
We consider initial determination of orbits for trans-neptunian objects (TNOs), a topical theme because of the rapidly growing TNO population and the challenges in recovering lost TNOs. We apply the method of initial phase-space ranging of orbits to the poorly observed TNOs. The rigorous a posteriori probability density of the TNO orbital elements is examined using a Monte Carlo technique by varying the TNO topocentric ranges corresponding to the observation dates. We can optionally adopt a Bayesian approach to select the region of phase space containing the most plausible orbits. This is accomplished by incorporating semimajor axes, eccentricities, inclinations, and absolute magnitudes of multi-apparition TNOs as a priori information. The resulting a posteriori distributions permit ephemeris and ephemeris uncertainty prediction for TNO recovery observations.
\end{abstract}

\section{Introduction}

After the discovery of the first Kuiper-belt objects nearly a decade ago, the orbital distribution of the Trans-Neptunian population has become a topic of growing interest among solar system studies. The TNO orbit determination problem has recently been approached by Bernstein and Khushalani (2000). They apply the fact that the gravitational accelerations can be treated as perturbations compared to the nearly inertial motion of the object. Their method appears very applicable to the case of poorly observed TNOs. Indeed, such less rigorous methods are worth developing since the projection of the complicated a posteriori probability density for the orbital elements into the sky plane often results in nearly linear distributions for the sky positions.

We applied the technique of statistical ranging of orbits, a new statistical orbit computation method devised by Virtanen, Muinonen, and Bowell (2001), for several poorly observed TNOs, of which we present the following examples.

\section{Results}

$1999 \mathrm{HB}_{12}$ had a 19-day arc (6 observations on two nights) following discovery, after which it was not observed until $\sim 1$ year later. We applied the ranging technique to the single-apparition arc from which no orbital elements could be 
obtained with standard orbit determination methods. The span of topocentric range, $[32,53]^{1} \mathrm{AU}$, resulted in a wide diversity of orbital elements: $a \in$ $\left.[25,150]^{2} \mathrm{AU}, e \in\right] 0,1\left[\right.$, and $i \in[15,165]^{\circ}$. The large number of high-eccentricity orbits indicates that near cometlike solutions are favoured without any a priori information. The orbital classification for $1999 \mathrm{HB}_{12}$ was equivocal; after the new observations, $1999 \mathrm{HB}_{12}$ is now known to be one of the distant TNOs but shortly after discovery, it showed only a mild tendency for the SKBO (Scattered Kuiperbelt object) class $(\sim 4 \%)$. To study the evolution of sky-plane uncertainties, we computed ephemeris distribution for the recovery date of March 28, 2000. For $1999 \mathrm{HB}_{12}$, a recovery strategy, very likely successful, could have been patterned on these predictions.

Our second example TNO, $1998 \mathrm{WW}_{31}$, was discovered in 1998 , followed up for $\sim 2$ months ( 8 observations on three nights), and is now considered lost. A broad variety of orbits $(a \in[40,150] \mathrm{AU}, e \in] 0,0.85])$ again resulted from the ranging method (topocentric range of $[44,47.5] \mathrm{AU}$ ) although the relative orientation of the orbit is rather well fixed $\left(i \in[5.5,8]^{\circ}, \Omega \in[236,238]^{\circ}\right)$. The distribution for current sky-plane uncertainty for $1998 \mathrm{WW}_{31}$ immediately suggests that planning of recovery attempts is useful. This asteroid has been classified as a classical TNO (i.e., not related to any mean motion resonance), which is also suggested by our results; $32 \%$ probability for classical TNO orbit.

\section{Conclusions}

Based on the studied TNO cases, it appears that the dynamical classification of Kuiper belt objects is highly uncertain for arcs shorter than two months. To make use of the Bayesian approach for TNOs, the current a priori distribution should be modified, taking into account that the known TNO population is only a small fraction of the one expected. For example, a two-dimensional (a,e)-distribution could be applied. The results for the recently recovered TNO $1999 \mathrm{HB}_{12}$ give strong indications that recovery observations for 'lost' objects can be attempted with reasonable observing schedules.

\section{References}

Bernstein, G., \& Khushalani B. 2000, AJ120, 3323

Virtanen, J., Muinonen, K., \& Bowell, E. 2001, Icarus, in press

\footnotetext{
${ }^{1}$ The small lower limit for $1999 \mathrm{HB}_{12}$ is now known to result from the fact that the object is a Scattered KBO (SKBO) which are typically discovered near perihelion between 34 and $36 \mathrm{AU}$ (near Neptune). Technically, distances beyond $\sim 50 \mathrm{AU}$ are suspect for new discoveries due to the current observing capabilities.
}

${ }^{2} \mathrm{~A}$ practical upper limit of $150 \mathrm{AU}$ has been adopted. 